Kohl: a Journal for Body and Gender Research

Vol. 2, No. 1 (Summer 2016)

\title{
Billboard Intervention
}

\author{
Tanja Van Deer \\ Saba Sadr \\ Salwa Mansour
}


Kohl 2.1

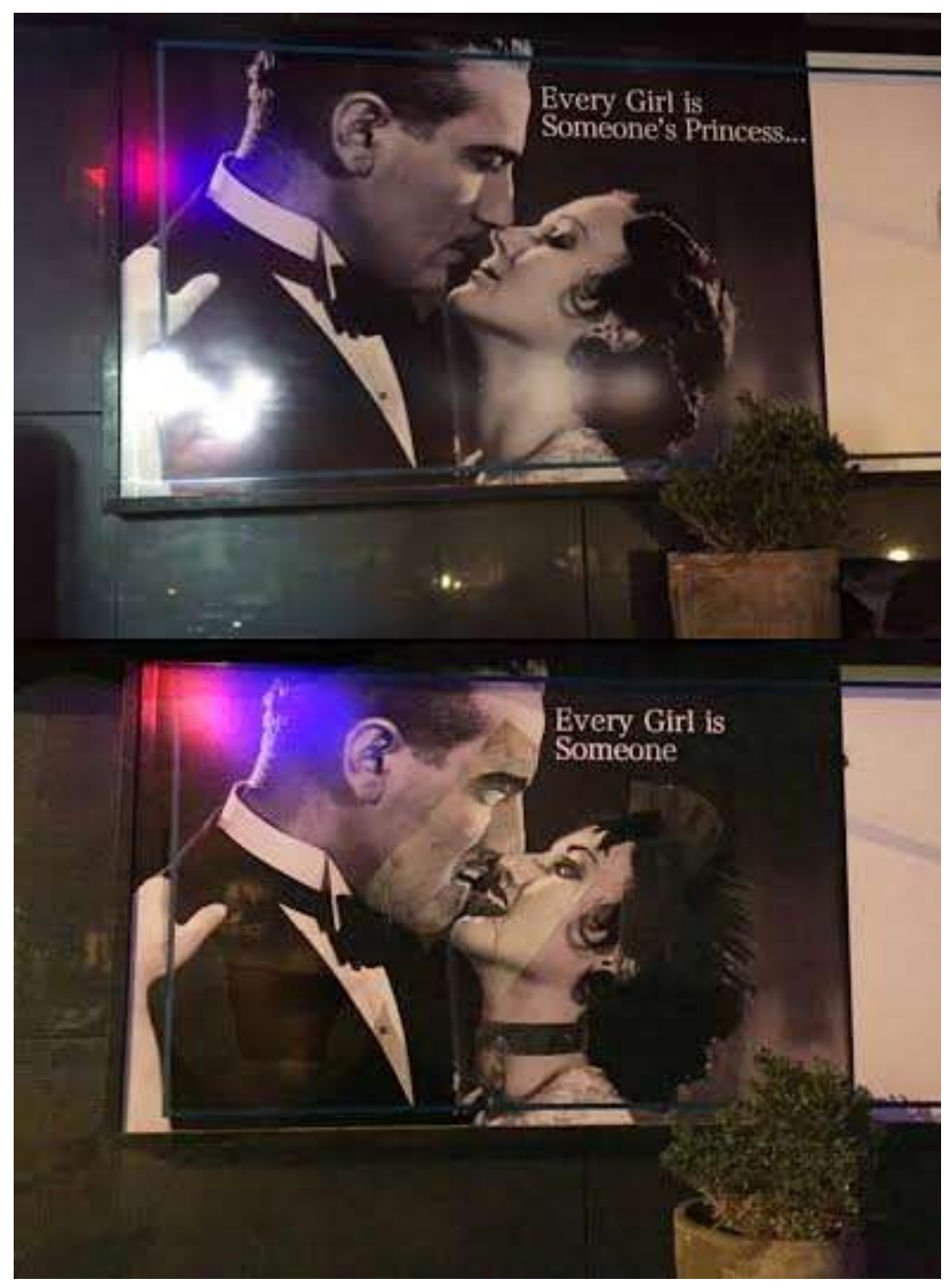

The original Damas Jewelry billboard barks sexist, heteronormative, and consumerist commands at passersby on Hamra street. Appalled by its arrogance, Tanja Van Deer and Saba Sadr plotted on how to intervene on the billboard through what is popularly known as "culture jamming," i.e. to visually and ideologically hijack an ad in order to subvert its original message. Tanja conceptualized the intervention. Saba artistically rendered the ideas. And Salwa Mansour aided both artists at successfully completing the late-night "coup." Their intent was to empower the once-passive female into an active agent who unapologetically revolted against the preordained parameters of her imprisonment. The high visibility of this billboard, they hoped, would leave a modest yet noticeable dent on an overwhelming sexist visual landscape. 